\title{
Continuous Mott Transition in a Two-Dimensional Hubbard Model
}

\author{
Yuki Yanag:* \\ Department of Physics, Faculty of Science and Technology, \\ Tokyo University of Science, Noda, Chiba 278-8510, Japan \\ Kazuo Ueda \\ Institute for Solid State Physics, University of Tokyo, Kashiwa, Chiba 277-8581, Japan
}

(Dated: February 26, 2018)

\begin{abstract}
We investigate nonmagnetic metal-insulator transition in the 1/5-depleted square lattice Hubbard model at half-filling within the 8-site cellular dynamical mean field theory. We find that a metalinsulator transition without any signatures of the first order transition, a continuous Mott transition, takes place in a certain range of parameters. The nature of the continuous Mott transition is nothing but a Lifshitz transition driven by the on-site Coulomb interaction. The renormalized matrix elements of hoppings and the spin-spin correlation functions reveal that physics of this transition is strong enhancement of the dimerization due to the non-local effects of electron-electron interaction.

PACS numbers: 71.30.+h, 71.10.Fd, 71.27.+a, 71.10.-w
\end{abstract}

\section{INTRODUCTION}

Metal-insulator transition (MIT) is one of the most intriguing phenomena in condensed matter physics 1 . Particularly, the MIT due to the strong Coulomb interaction, known as the Mott transition, has attracted much attention and a considerable number of theoretical and experimental studies have been devoted to elucidate generic features of the Mott transition. According to these studies, it has been clarified that properties of the Mott transition strongly depend on the lattice structure, such as connectivity and geometrical frustration. The Mott transition on non-Bravais lattices is especially interesting ${ }^{2}-\underline{-}$. In non-Bravais lattices, the crystallographic unit cell contains at least two distinct sublattices and the resulting multi-band electronic structures bring various possibilities into the Mott transition.

One typical example is the honeycomb lattice Hubbard model in which the Dirac cones are located on the Fermi level at half-filling. Possibility of the Mott transition without any signatures of the first order transition, the continuous Mott transition, and a spin liquid ground state without geometrical frustration have been discussed in the literature $e^{2.3}$ although the recent state-ofthe-art quantum Monte Carlo (QMC) simulation is negative for the nonmagnetic insulating (NI) ground state $\mathrm{e}^{3}$. Another example is the bilayer Hubbard model in which the two square lattice Hubbard layers are coupled with each other through an inter-layer hopping $t_{\perp}$. The model has been studied by the cellular dynamical mean field theory (CDMFT) with $\sqrt{2} \times \sqrt{2} \times 2$-site cluster ${ }^{4}$ and it has been shown that for larger values of $t_{\perp}$, the antiferromagnetic insulating (AFI) phase is suppressed and a nonmagnetic metallic state appears between the AFI and NI phases despite the perfect nesting of the Fermi surfaces. It is natural to expect that this result indicates the presence of a continuous Mott transition. According to the recent variational Monte Carlo (VMC) study ${ }^{5}$, however, the AFI phase extends for the larger inter-layer hopping regime and the continuous Mott transition is masked by the AFI phase. Possibility of the nonmagnetic metallic ground state without geometrical frustration and the continuous Mott transition have been discussed also in the ionic Hubbard model where the staggered potential $\pm \Delta$ is applied on the $\mathrm{A}$ or $\mathrm{B}$ sublattices. The studies by the single-site dynamical mean field theory (DMFT) ${ }^{6}$ and by the QMC simulation ${ }^{7}$ give positive results, while the CDMFT study ${ }^{8}$ a negative one. In any case, it is still controversial whether the continuous Mott transition and the metallic ground state without geometrical frustration is possible or not.

In the present paper, we show that the Hubbard model on the $1 / 5$-depleted square lattice ${ }^{\underline{\underline{9}}} \underline{\underline{13}}$ at half-filling exhibits the continuous Mott transition in the paramagnetic phase, where the model in the strong coupling limit is considered to be a minimal model for $\mathrm{CaV}_{4} \mathrm{O}_{9} \frac{9}{9}$ which is a typical example of spin-gapped systems. The $1 / 5$ depleted square lattice is a kind of non-Bravias lattice in which there are four distinct sublattices $(A-D)$ in the unit cell [see Fig. 10 (a)]. We study the MIT in the paramagnetic phase of this model with use of the CDMFT ${ }^{14,15}$ combined with the continuous-time auxiliary field quantum Monte Carlo method (CT-AUX)16,17. It is shown that in this model, the nonmagnetic continuous Mott transition is driven by the promotion of dimerization due to the non-local electron correlation effects.

\section{1/5-DEPLETED SQUARE LATTICE HUBBARD MODEL AND CDMFT}

We consider the Hubbard model on the $1 / 5$-depleted square lattice,

$$
H=\sum_{i, j, \alpha, \beta} t_{i, \alpha, j, \beta} c_{i \alpha \sigma}^{\dagger} c_{j \beta \sigma}+U \sum_{i \alpha} n_{i \alpha \uparrow} n_{i \alpha \downarrow}
$$



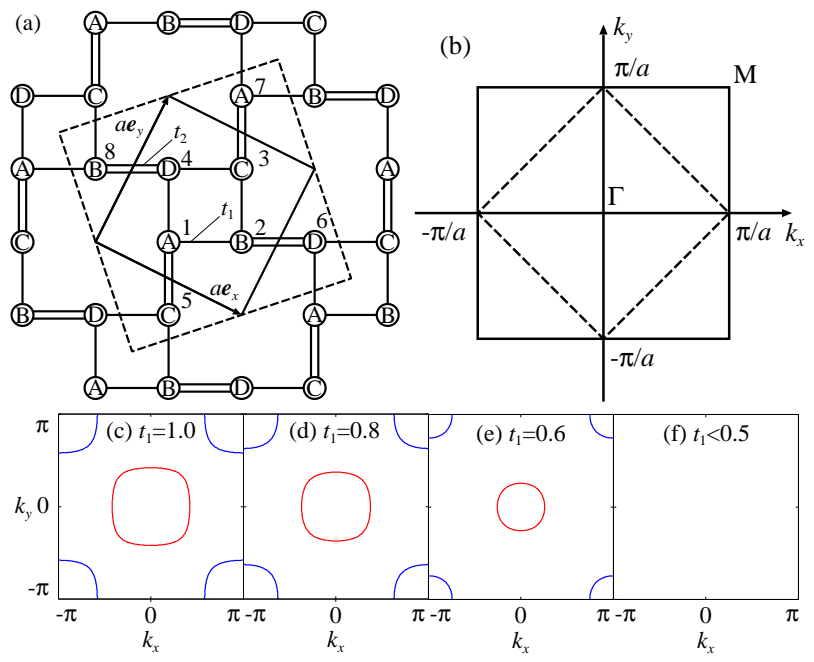

FIG. 1. (Color online) (a) The schematic of the $1 / 5$-depleted square lattice. The single and double solid lines represent the intra-plaquette and intra-dimer hoppings $t_{1}$ and $t_{2}$, respectively. The solid and dashed squares represent the crystallographic unit cell and the 8-site supercell, respectively. (b) The first Brillouin zone (solid square) and the reduced Brillouin zone (dashed square) corresponding to the 8-site supercell. (c)-(f) Fermi surfaces for several values of $t_{1}$.

where $c_{i \alpha \sigma}^{(\dagger)}$ annihilates (creates) an electron with spin $\sigma$ on the sublattice $\alpha$ at the unit cell $i, n_{i \alpha \sigma}=c_{i \alpha \sigma}^{\dagger} c_{i \alpha \sigma}$, $t_{i, \alpha, j, \beta}$ and $U$ represent the nearest neighbor hopping integrals and the on-site Coulomb interaction, respectively. As schematically shown in Fig. 1] (a), $t_{i, \alpha, j, \beta}=t_{1}\left(t_{2}\right)$ on plaquette- (dimer-) bonds, where we refer to $t_{1}\left(t_{2}\right)$ as the intra-plaquette (-dimer) hopping, here and hereafter. We treat $t_{1}$ and $t_{2}$ as independent parameters because they are not geometrically equivalent in the $1 / 5$-depleted square lattice. In the present study, we concentrate on the case of $t_{1}<t_{2}$ and the energy is measured in units of $t_{2}$ hereafter. According to our previous studies ${ }^{12,13}$, single electron properties of the model are significantly different depending on values of $t_{1}$ and $t_{2}$. For $t_{1}<t_{2}$, there are four energy bands composed of two valence and two conduction bands and the bottom (top) of the conduction (valence) bands is located on the energy $-2 t_{1}+t_{2}$ $\left(2 t_{1}-t_{2}\right)$ at the $\Gamma-(M-)$ point and the total band width $W=4 t_{1}+2 t_{2} \leq 6 t_{2}$, where we note that the first Brillouin zone is usual square shape as shown in Fig. 1 (b). Therefore, for $0.5 t_{2} \leq t_{1}<t_{2}$, the noninteracting ground state is (semi-)metallic and there is a electron (hole) pocket around the $\Gamma_{-}\left(M_{-}\right)$point as shown in Figs. 1 (c)-(e). With decreasing $t_{1}$, the Fermi surfaces shrink and finally vanish at $t_{1}=0.5 t_{2}$ as shown in Figs. 1] (c)(f). Thus, the noninteracting ground state is insulating for $t_{1}<0.5 t_{2}$.

To study effects of electron correlation in the $1 / 5$ depleted square lattice Hubbard model at half-filling, we use the CDMFT ${ }^{14,15}$, one of the cluster extensions of the DMFT. In the CDMFT, the original lat- tice problem is mapped onto an effective cluster problem with open boundary conditions embedded in a selfconsistent electronic bath. In our previous conference presentation 13 , we have reported preliminary results by using the CDMFT with a 4-site cluster including 2dimers. The cluster has much lower symmetry than the original problem and cluster size dependence has not been investigated. In addition, for $t_{1} \sim t_{2}$, the results of the Heisenberg mode ${ }^{9}-11$ indicate competition between the dimer-singlet and the plaquette-singlet states ${ }^{9}-11$. Therefore, the CDMFT study with a larger cluster including both plaquettes and dimers is highly desired. In the present study, we use the 8-site cluster depicted in Fig. 1 (a). In the CDMFT, the bath Green's function $\hat{\mathcal{G}}\left(i \varepsilon_{n}\right)$, the cluster Green's function $\hat{G}\left(i \varepsilon_{n}\right)$ and the cluster self-energy $\hat{\Sigma}\left(i \varepsilon_{n}\right)$ are self-consistently determined through the following procedure, where $\hat{\mathcal{G}}\left(i \varepsilon_{n}\right), \hat{G}\left(i \varepsilon_{n}\right)$ and $\hat{\Sigma}\left(i \varepsilon_{n}\right)$ have $8 \times 8$ matrix forms and $\varepsilon_{n}=(2 n+1) \pi T$ is the fermionic Matsubara frequency. Initially, one guess a bath Green's function $\hat{\mathcal{G}}\left(i \varepsilon_{n}\right)$. Next, we solve the the effective cluster problem characterized by $\hat{\mathcal{G}}\left(i \varepsilon_{n}\right)$ with use of the CT-AUX ${ }^{16,17}$ to obtain $\hat{G}\left(i \varepsilon_{n}\right)$ and $\hat{\Sigma}\left(i \varepsilon_{n}\right)$. Then, a new bath Green's function can be computed via the CDMFT self-consistency condition,

$$
\begin{aligned}
& \hat{G}\left(i \varepsilon_{n}\right)=\sum_{\tilde{\boldsymbol{k}} \in \mathrm{RBZ}}\left\{\left(i \varepsilon_{n}+\mu\right) \hat{1}-\hat{t}(\tilde{\boldsymbol{k}})-\hat{\Sigma}\left(i \varepsilon_{n}\right)\right\}^{-1} \\
& \left\{\hat{\mathcal{G}}\left(i \varepsilon_{n}\right)\right\}^{-1}=\left\{\hat{G}\left(i \varepsilon_{n}\right)\right\}^{-1}+\hat{\Sigma}\left(i \varepsilon_{n}\right)
\end{aligned}
$$

where $\tilde{\boldsymbol{k}}$-integration is performed over the reduced Brillouin zone (RBZ) shown in Fig. 1 (b) and $\hat{t}(\tilde{\boldsymbol{k}})$ is the Fourier-transformed hopping matrix for the 8-site cluster. This iterative procedure is performed until the converged results are obtained. It is noted that the chemical potential is $\mu=0$ at the half-filled case studied in this paper because of the bipartite nature of the $1 / 5$-depleted square lattice $\frac{12,13}{}$. In the present study, to investigate the correlation effects on the phase without any symmetry breaking, we assume a paramagnetic solution in numerical calculations and set $T=0.1 t_{2}$. However, we have confirmed that the results as will be shown below do not change qualitatively down to $T=0.05 t_{2}$.

\section{CONTINUOUS MI TRANSITION}

First, in Fig. 2, we show results on the double occupancy $d=\left\langle n_{i \alpha \uparrow} n_{i \alpha \downarrow}\right\rangle$ as a function of $U$ for $0.1 t_{2} \leq$ $t_{1} \leq t_{2}$. It is observed that $d$ decreases monotonically with increasing $U$ and there is no discontinuous jump up to $U=6 t_{2}$. This indicates absence of the first order Mott transition as far as $t_{1} \leq t_{2}$ in contrast to the case of the ordinary Hubbard model on the square lattice ${ }^{18}$. It is noteworthy that $d$ for $t_{1}>0.5 t_{2}$ exhibits qualitatively different behavior from that for $t_{1}<0.5 t_{2}$. For $t_{1}<0.5 t_{2}, U$-dependence of $d$ is always concave upward, 


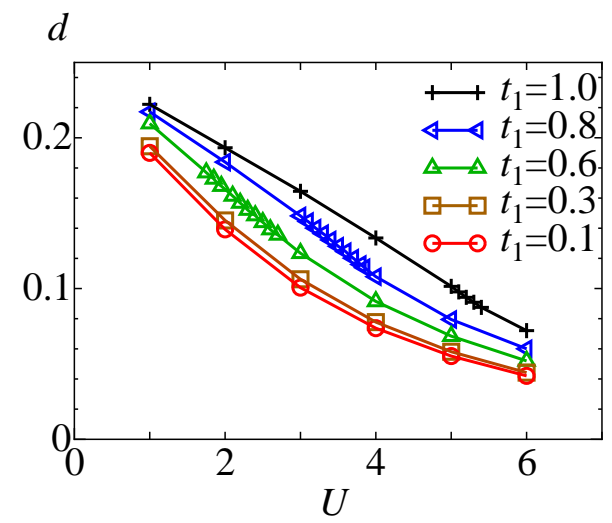

FIG. 2. (Color online) $U$-dependence of the double occupancy $d$ for several values of $t_{1}$.

while for $t_{1}>0.5 t_{2}$, it changes from concave downward to upward at a certain value of $U$, signaling the MIT as will be mentioned later.

To further investigate the correlation effects, $\varepsilon_{n^{-}}$ dependence of the local self-energy $\operatorname{Im} \Sigma_{11}\left(i \varepsilon_{n}\right)$ and the intra-dimer self-energy $\operatorname{Re} \Sigma_{15}\left(i \varepsilon_{n}\right)$ for several values of $U$ at $t_{1}=0.3 t_{2}$ and $t_{1}=0.8 t_{2}$ are shown in Fig. 3. We note that for $t_{1}=0.3 t_{2}$, the noninteracting ground state is a band insulator, while for $t_{1}=0.8 t_{2}$, that is a metal as mentioned before. For both cases, the local components $\operatorname{Im} \Sigma_{11}\left(i \varepsilon_{n}\right)$ and the intra-dimer components $\operatorname{Re} \Sigma_{15}\left(i \varepsilon_{n}\right)$ are enhanced with increasing $U$. Especially, the enhancement of $\operatorname{Re} \Sigma_{15}\left(i \varepsilon_{n}\right)$ in the low energy region is significant, which is the key to understand the MIT in the present model as will be shown later. It has also been observed that the local components $\operatorname{Im} \Sigma_{11}\left(i \varepsilon_{n}\right)$ have no singularities and are proportional to $\varepsilon_{n}$ in the low energy region. The analytic behavior of $\hat{\Sigma}\left(i \varepsilon_{n}\right)$ allows us to expand $\hat{\Sigma}\left(i \varepsilon_{n}\right)$ with respect to $\varepsilon_{n}$ for $\varepsilon_{n} \sim 0$, i.e., the quasiparticle picture can be used to describe the electronic states up to relatively large value of $U=6 t_{2}$. Then, the quasiparticle cluster Green's function $\hat{\tilde{G}}(\omega)$ is written as
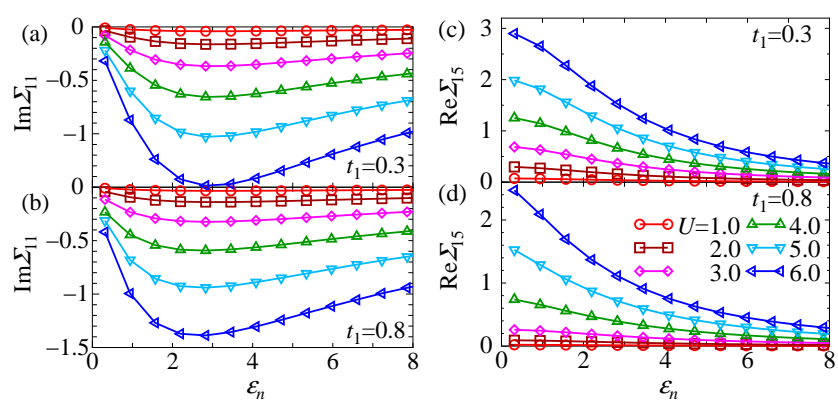

FIG. 3. (Color online) (a) and (b) $\varepsilon_{n}$-dependence of the imaginary part of the local self-energy $\operatorname{Im} \Sigma_{11}\left(i \varepsilon_{n}\right)$, (c) and (d) those of the real part of the intra-dimer self-energy $\operatorname{Re} \Sigma_{15}\left(i \varepsilon_{n}\right)$ for $t_{1}=0.3 t_{2}$ and $0.8 t_{2}$.
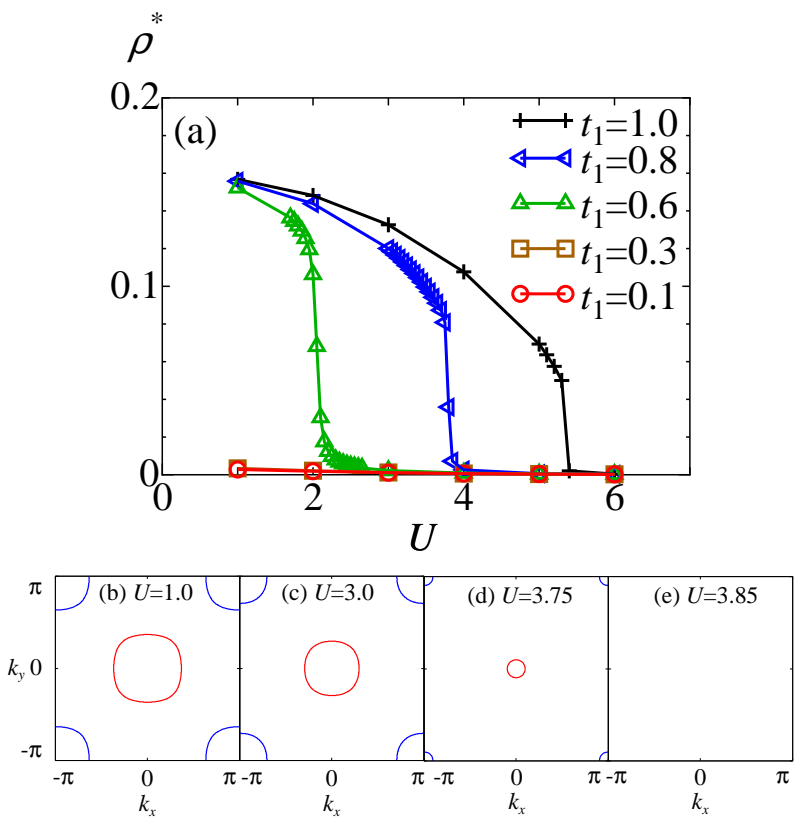

FIG. 4. (Color online) (a) $U$-dependence of the quasiparticle DOS $\rho^{*}$. It is noted that the small residual DOS is due to the Lorenzian broadening in the numerical calculation. (b)-(e) Fermi surfaces for several values of $U$ at $t_{1}=0.8 t_{2}$.

follows,

$$
\begin{aligned}
\hat{G}(\omega) & \sim \hat{\tilde{G}}(\omega)=\sum_{\tilde{\boldsymbol{k}} \in \mathrm{RBZ}} \hat{Z}^{\frac{1}{2}}\left[(\omega+\mu) \hat{1}-\hat{t}^{*}(\tilde{\boldsymbol{k}})\right]^{-1} \hat{Z}^{\frac{1}{2}} \\
\hat{t}^{*}(\tilde{\boldsymbol{k}}) & =\hat{Z}^{\frac{1}{2}}\left[\hat{t}(\tilde{\boldsymbol{k}})+\frac{\hat{\Sigma}(i \pi T)+\hat{\Sigma}^{\dagger}(i \pi T)}{2}\right] \hat{Z}^{\frac{1}{2}} \\
\hat{Z} & =\left[\hat{1}-\frac{\hat{\Sigma}(i \pi T)-\hat{\Sigma}^{\dagger}(i \pi T)}{2 i \pi T}\right]^{-1}
\end{aligned}
$$

where $\hat{t}^{*}(\tilde{\boldsymbol{k}})$ and $\hat{Z}$ are the Fourier-transformed renormalized hopping matrix in the cluster and the renormalization factor, respectively. The $U$-dependence of the quasiparticle density of states (DOS) at the Fermi level $\rho^{*}=\frac{1}{\pi} \operatorname{Im} \hat{\tilde{G}}_{11}(0)$ is shown in Fig. 4. On one hand, for $t_{1} \leq 0.5 t_{2}, \rho^{*}$ is always 0 and the insulating ground state is realized irrespective of the values of $U$. On the other hand, for $0.6 t_{2} \leq t_{1} \leq t_{2}, \rho^{*}$ monotonically decreases with increasing $U$ and vanishes at a certain critical value $U=U_{c}$. This clearly shows that the MIT takes place at $U=U_{c}$ without any signatures of the first order Mott transition, such as, discontinuous jumps in $U$ - $d$ curves and singularities in $\hat{\Sigma}\left(i \varepsilon_{n} \sim 0\right)$ as mentioned before. In other words, the Mott transition in the present model is continuous.

To gain more insight about the MIT, we have calculated the Fermi surfaces of the interacting system. The Fermi surface in the interacting system is defined as lines 


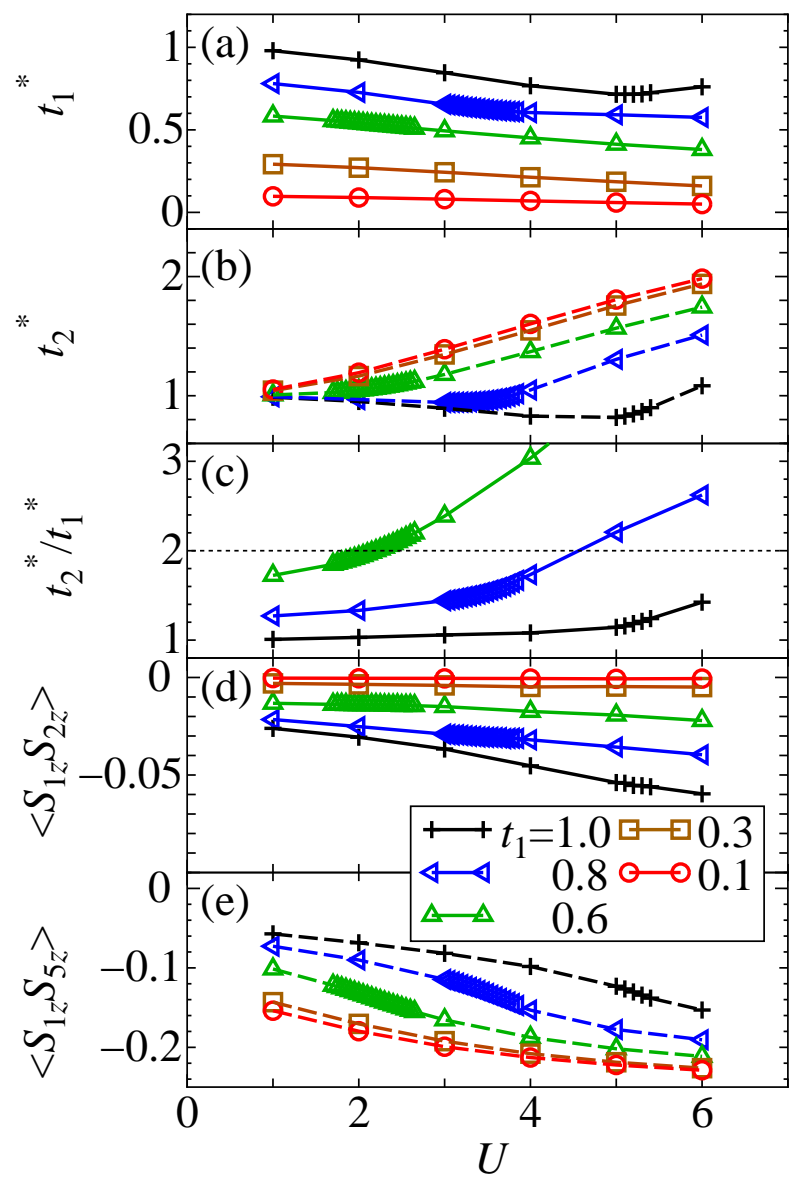

FIG. 5. (Color online) $U$-dependence of (a) the effective cluster hopping $t_{1}^{*}$, (b) $t_{2}^{*}$, (d) the relative value $t_{2}^{*} / t_{1}^{*}$, (d) the intra-plaquette spin-spin correlation $\left\langle S_{1 z} S_{2 z}\right\rangle$ and (e) intradimer spin-spin correlation $\left\langle S_{1 z} S_{5 z}\right\rangle$ for several values of $t_{1}$.

of the $\boldsymbol{k}$-points where the following equation is satisfied:

$$
\operatorname{det}\left[\hat{H}_{0}(\boldsymbol{k})+\frac{\hat{\Sigma}^{\mathrm{L}}(\boldsymbol{k}, i \pi T)+\hat{\Sigma}^{\mathrm{L} \dagger}(\boldsymbol{k}, i \pi T)}{2}\right]=0,
$$

where the Fourier representation of the kinetic part of the Hamiltonian $\hat{H}_{0}(\boldsymbol{k})$ and the $\boldsymbol{k}$-dependent lattice selfenergy $\hat{\Sigma}^{\mathrm{L}}\left(\boldsymbol{k}, i \varepsilon_{n}\right)$ have $4 \times 4$ matrix forms whose elements are labeled by the four distinct sublattices $\alpha, \beta(=A-D)$ contained in the crystallographic unit cell. It should be noted that momentum $\boldsymbol{k}$ is defined in the first Brillouin zone unlike $\tilde{\boldsymbol{k}}$ in eqs. (2), (4) and (5). Since in the CDMFT, the translational symmetry is violated, to obtain the $\boldsymbol{k}$-dependent lattice self-energy $\hat{\Sigma}^{\mathrm{L}}\left(\boldsymbol{k}, i \varepsilon_{n}\right)$, we perform the periodization,

$$
\Sigma_{\alpha \beta}^{\mathrm{L}}\left(\boldsymbol{k}, i \varepsilon_{n}\right)=\frac{1}{2} \sum_{m, m^{\prime}}^{\prime} \Sigma_{m m^{\prime}}\left(i \varepsilon_{n}\right) e^{-i \boldsymbol{k} \cdot\left(\boldsymbol{r}_{m}-\boldsymbol{r}_{m}^{\prime}\right)},
$$

where $\boldsymbol{r}_{1}=\boldsymbol{r}_{2}=\boldsymbol{r}_{3}=\boldsymbol{r}_{4}=(0,0), \boldsymbol{r}_{5}=(0,-a), \boldsymbol{r}_{6}=$ $(a, 0), \boldsymbol{r}_{7}=(0, a)$ and $\boldsymbol{r}_{8}=(-a, 0)$ and the prime on the sum restricts the summation over $m$ and $m^{\prime}$ to the sites belonging to the sublattices specified by $\alpha$ and $\beta$, respectively, e.g. $m=1$ and 7 for $\alpha=A$ [see Fig. 1 (a)].

Change of the Fermi surface as a function of $U$ is shown in Figs. 4 (b)-(e). It is observed that both the electron and the hole pockets shrink with increasing $U$ and finally vanish at $U=U_{c}$ in a similar way as the Fermi surface evolution with decreasing $t_{1}$ in the noninteracting system which is shown in Figs. 11 (c)-(f). This suggests that the MIT for $0.5 t_{2} \leq t_{1}<t_{2}$ is nothing but a Lifshitz transition ${ }^{19}$. It is worth mentioning that a conventional Lifshitz transition is topological change of Fermi surfaces driven by variations of one particle parameters 19 , such as a chemical potential or a transfer integral, while the one we find in the present model is driven by the electron correlation effects without any symmetry breaking.

Here, we show the renormalized cluster hopping $\hat{t}^{*}=$ $\sum_{\tilde{\boldsymbol{k}} \in \mathrm{RBZ}} \hat{t}^{*}(\tilde{\boldsymbol{k}})$ in Figs. 5 (a)-(c). Since in the noninteracting case, the ground state properties are determined by the values of $t_{1}$ and $t_{2}$ and the MIT takes place at $t_{1}=0.5 t_{2}$ as mentioned before, the renormalized cluster hopping $\hat{t}^{*}$ can give us an intuitive physical picture about the MIT. Figs. 5 (a) and (b) shows the $U$-dependence of the renormalized intra-plaquette and intra-dimer hoppings $t_{1}^{*} \equiv t_{12}^{*}$ and $t_{2}^{*} \equiv t_{15}^{*}$, respectively. We find that with increasing $U$, the effective intra-plaquette hopping $t_{1}^{*}$ decreases due to the effects of the renormalization factor $\hat{Z}$ whose eigen values are smaller than 1 . In contrast, the effective intra-dimer hopping $t_{2}^{*}$ decreases more slowly than $t_{1}^{*}$ up to a slightly smaller value than $U_{c}$ and starts to increase with further increasing $U$. Therefore, the resulting $t_{2}^{*} / t_{1}^{*}$ monotonically increases with increasing $U$ as shown in Fig. [5 (c). This indicates that the electron correlation effects significantly promote the dimerization and lead the system to the insulating state. Therefore, the insulating state realized for $U \geq U_{c}$ is referred to as the dimer-insulator. It is noted that the dimerization induced by the electron correlation is due to the effects of the off-diagonal elements of the self-energy shift $\frac{\hat{\Sigma}(i \pi T)+\hat{\Sigma}^{\dagger}(i \pi T)}{2}$ which are the non-local effects neglected in the single-site DMFT and whose intra-dimer component is significantly enhanced in the present case as shown in Figs. 3 (c) and (d).

The enhancement of the dimerization is also observed in the two-particle quantities such as the spin-spin correlation functions $\left\langle S_{m z} S_{m^{\prime} z}\right\rangle$. The nearest neighbor spinspin correlations $\left\langle S_{m z} S_{m^{\prime} z}\right\rangle$ are shown in Figs. 5 (d) and (e). It is found that both the intra-plaquette component $\left\langle S_{1 z} S_{2 z}\right\rangle$ and the intra-dimer component $\left\langle S_{1 z} S_{5 z}\right\rangle$ are negative, that is, the nearest neighbor spin-spin correlations have tendency towards spin-singlet. With increasing $U$, the singlet correlation on a plaquette develops only slightly, while that on a dimer is largely enhanced. We find that the characteristic features observed in $U$-dependence of the double occupancy $d$ are more clearly seen in that of the intra-dimer spin-spin correlation $\left\langle S_{1 z} S_{5 z}\right\rangle$. In particular, the sign-reversal of the curvature across the critical point $U_{c}$ in $U-\left\langle S_{1 z} S_{5 z}\right\rangle$ 


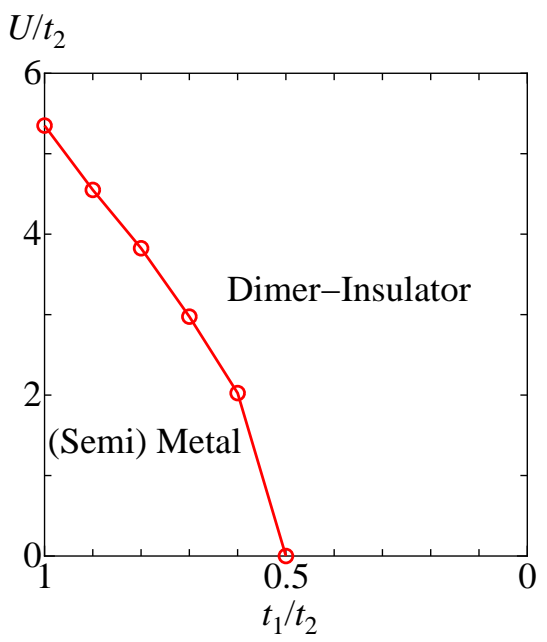

FIG. 6. (Color online) Phase diagram on $t_{1}-U$ plane for $T=$ $0.1 t_{2}$.

curves is more pronounced compared with $U$ - $d$ curves for $t_{1}>0.5 t_{2}$.

Finally, we show the phase diagram on $t_{1}-U$ plane in Fig. 6. For $t_{1} \geq 0.5 t_{2}$, the continuous transition between the (semi)metal and the dimer-insulator takes place at $U=U_{c}$ as mentioned before, where $U_{c}$ is determined as the values at which the Fermi surfaces vanish. The phase diagram is very similar to that obtained previously by the 4-site CDMFT study $\underline{\underline{13}}$ and the cluster-size dependence is small. It should be noted that the critical value $U_{c}$ is always smaller than the total bandwidth $W=4 t_{1}+2 t_{2}$. On the other hand, for $t_{1}<0.5 t_{2}$, with increasing $U$, the electronic states smoothly vary from the noninteracting limit to the strong coupling regime. It is clear that the dimer-insulator is adiabatically connected to the band insulator.

\section{SUMMARY AND DISCUSSION}

In summary, we have studied the nonmagnetic MIT in the Hubbard model on the 1/5-depleted square lattice at half-filling for the case that the intra-dimer hopping $t_{2}$ is larger than the intra-plaquette one $t_{1}$ by using the 8-site cellular dynamical mean field theory combined with the continuous-time auxiliary field quantum Monte Carlo method. We have found that for $0.5 t_{2}<t_{1}<t_{2}$, the Coulomb interaction drives the MIT without any signatures of the first order transition, that is, the Mott transition is continuous in contrast to the case of the ordinary square lattice Hubbard model in which the first order Mott transition takes place. We have also shown that the continuous Mott transition is nothing but a Lifshitz transition $\frac{19}{}$ and is originated from the significant enhancement of the dimerization by the non-local electron correlation effects ${ }^{20,21}$. These results are consistent with the CDMFT studies discussed for the mechanism of the MIT in $\mathrm{NaV}_{2} \mathrm{O}_{5}{ }^{20}$ and $\mathrm{Ti}_{2} \mathrm{O}_{3}{ }^{21}$ but the correlationinduced dimerization is more pronounced in the present case than the above two cases in which the inter-site interaction $V$ is essential for the formation of the sufficiently large band gap. Moreover, it is found that the dimer-insulator is adiabatically connected to the band insulator.

Finally, we comment on the stability of the nonmagnetic continuous Mott transition against the antiferromagnetism. In the present study, our numerical calculations are performed within the paramagnetic phase and the possibility of the antiferromagnetism has not been considered so far. Of course, the bipartite nature of the $1 / 5$-depleted square lattice leads to the perfect nesting of the Fermi surfaces for $t_{1} \geq 0.5 t_{2}$ as shown in Figs. 1 (c)-(f) and the resulting bare staggered susceptibility diverges at $T=0$ although the singularity is weaker than that in the ordinary square lattice. Therefore, whether the quantum fluctuations remove this instability or not is the central issue. Since in the $1 / 5$-depleted square lattice, the connectivity $z$ is small $(z=3)$ and furthermore, the lattice can be viewed as a collection of local units which naturally accommodate local singlets, such as dimers or plaquettes, the effects of quantum fluctuations are expected to be strong. In fact, according to the results of the Heisenberg model on the 1/5-depleted square lattice ${ }^{9}-11$, the antiferromagnetism is confined only in the very small parameter region $0.77 t_{2} \lesssim t_{1} \lesssim 1.03 t_{2} \underline{10,11}$. On the other hand, the recent determinant QMC study of the present model predicts the AFI ground state for the intermediate correlation regime ${ }^{22}$. Therefore, the nonmagnetic metallic states and the continuous Mott transition observed in the present study is considered to be the metastable state and would be masked by the AFI state if one allows magnetic solutions. However, because the quantum fluctuations in the present model are strong as mentioned above, the nonmagnetic metallic states and the continuous Mott transition can be stabilized by introducing a weak geometrical frustration.

\section{ACKNOWLEDGMENTS}

A part of the computation in this work has been done using the facilities of the Supercomputer Center at ISSP, University of Tokyo. This work has been supported by a Grant-in-Aid for Scientific Research on Innovative Areas Heavy Electrons (No. 20102008) and (C) (No. 25400357). 
70, 1039 (1998).

2 Z. Y. Meng, T. C. Lang, S. Wessel, F. F. Assaad and A. Muramatsu, Nature (London) 464, 847 (2010).

3 S. Sorella, Y. Otsuka and S. Yunoki, Sci. Rep. 2, 992 (2012).

4 S. S. Kancharla and S. Okamoto, Phys. Rev. B 75, 193103 (2007).

5 R. Rüger, L. F. Tocchio, R. Valentí and C. Gros, New J. Phys. 16033010 (2014).

6 A. Garg, H. R. Krishnamurthy and M. Randeria, Phys. Rev. Lett. 97, 046403 (2006).

7 N. Paris, K. Bouadim, F. Hebert, G. G. Batrouni and R. T. Scalettar, Phys. Rev. Lett. 98, 046403 (2007).

8 S. S. Kancharla and E. Dagotto, Phys. Rev. Lett. 98, 016402 (2007).

9 K. Ueda, H. Kontani, M. Sigrist and P. A. Lee, Phys. Rev. Lett. 76, 1932 (1996).

10 M. Troyer, H. Kontani, K. Ueda, Phys. Rev. Lett. 76, 3822 (1996).

11 M. Troyer, M. Imada, K. Ueda, J. Phys. Soc. Jpn. 66, 2957 (1997).

12 Y. Yamashita, M. Tomura, Y. Yanagi and K. Ueda, Phys.
Rev. B 88, 195104 (2013).

13 Y. Yanagi and K. Ueda, to be published in JPS Conf. Proc.

14 G. Kotliar, S. Y. Savrasov, G. Pálsson and G. Biroli, Phys. Rev. Lett. 87, 186401 (2001).

15 T. Maier, M. Jarrell, T. Pruschke and M. H. Hettler, Rev. Mod. Phys. 77, 1027 (2005).

16 E. Gull, P. Werner, O. Parcollet and M. Troyer, Europhys. Lett. 82, 57003 (2008).

17 E. Gull, A. J. Millis, A. I. Lichtenstein, A. N. Rubtsov, M. Troyer and P. Werner, Rev. Mod. Phys. 83, 349 (2011).

18 H. Park, K. Haule and G. Kotliar, Phys. Rev. Lett. 101, 186403 (2008).

19 I. M. Lifshitz, Sov. Phys. JETP 11, 1130 (1960).

20 V. V. Mazurenko, A. I. Lichtenstein, M. I. Katsnelson, I. Dasgupta, T. Saha-Dasgupta and V. I. Anisimov, Phys. Rev. B 66, 081104 (2002).

21 A. I. Poteryaev, A. I. Lichtenstein, and G. Kotliar, Phys. Rev. Lett. 93, 086401 (2004).

22 E. Khatami, R. R. P. Singh, W. E. Pickett, and R. T. Scalettar, arXiv:1404.3731. 